\title{
Nouvelles spectroscopies Raman X du carbone pour les matériaux anciens
}

\author{
Uwe Bergmann(1) (bergmann@slac.stanford.edu), Rafaella Georgiou(2), \\ Pierre Gueriau ${ }^{(2)}$, Jean-Pascal Rueff ${ }^{(3)}$ et Loïc Bertrand ${ }^{(2)}$ \\ (loic.bertrand@synchrotron-soleil.fr) \\ (1) Stanford PULSE Institute, SLAC National Accelerator Center, Menlo Park, CA, USA \\ (2) IPANEMA (USR3461 CNRS), Université Paris-Saclay, Site du Synchrotron SOLEIL, \\ BP 48, 91192 Gif-sur-Yvette \\ (3) Synchrotron SOLEIL, BP 48, 91192 Gif-sur-Yvette
}

L'identification des composés à base de carbone, bien que difficile, est une source d'information essentielle dans de nombreuses études archéologiques

et paléontologiques.

\section{La diffusion Raman de rayons $\mathrm{X}$}

est une méthode de spectroscopie sur synchrotron qui permet d'identifier des signatures organiques, de retracer l'origine chimique des systèmes étudiés et de comprendre l'altération des composés organiques dans le temps.

\section{Cette technique, conduite} de manière non destructive, dans l'air, avec une sensibilité en profondeur afin de fournir des informations non compromises par la contamination superficielle, surmonte ainsi plusieurs contraintes fondamentales à la caractérisation des matériaux organiques anciens.
Les matériaux du patrimoine culturel et d'histoire naturelle sont souvent riches en carbone, élément qui se présente sous différentes formes moléculaires. Des spectroscopies vibrationnelles (reposant sur l'excitation des liaisons chimiques), comme les spectroscopies infrarouge ou Raman optique, ou bien électroniques comme la spectroscopie d'absorption de rayons $\mathrm{X}$, permettent d'identifier ces différentes formes de carbone.

\section{Difficile analyse des formes chimiques du carbone}

Les méthodes disponibles pour l'identification des composés organiques présentent des contraintes très fortes quand on les applique à l'étude des matériaux anciens.

Les méthodes invasives dites "séparatives ", comme la chromatographie, sont employées pour identifier avec grande précision les molécules organiques anciennes dans les échantillons pour lesquels un microprélèvement est autorisé ; cependant, aucune information spatiale à petite échelle ne peut être obtenue par ces méthodes.

La spectroscopie infrarouge à transformée de Fourier, la microspectroscopie Raman optique, la diffraction de rayons $\mathrm{X}$ et la spectroscopie de masse à ionisation secondaire (ToF-SIMS) sont souvent utilisées pour identifier les composés organiques et documenter la nature chimique et l'organisation des matériaux carbonés modernes, par analyse ponctuelle ou en imagerie. Pour les matériaux anciens, en plus de l'intense contribution de la fluorescence qui entrave souvent l'interprétation du signal Raman, la principale limitation des spectroscopies ToF-SIMS, Raman optique et infrarouge est qu'elles ne sondent que les premiers micromètres de matière à la surface. Elles exacerbent donc la contribution de la contamination superficielle, à moins de procéder par examen de coupes transverses, souvent difficiles à produire, et qui engendrent parfois une détérioration de l'état de la surface... que l'on est en train d'analyser.

Les rayons $\mathrm{X}$ ont assez d'énergie pour éjecter les électrons de cœur de l'atome dont les énergies de liaison identifient chaque élément de façon unique. La spectroscopie d'absorption de rayons $\mathrm{X}$ dite "proche du seuil [d'absorption]", désignée par son acronyme anglais "XANES », sonde les niveaux d'énergie des orbitales libres à proximité d'un tel seuil d'absorption, notamment celles correspondant à des liaisons chimiques. La spectroscopie XANES est donc couramment employée pour l'identification précise des composés chimiques dans lesquels se trouve un élément choisi dans un échantillon. Cette "spéciation chimique "s'applique par exemple aux métaux de transition, comme le manganèse, le fer ou le cuivre. Les progrès réalisés en termes d'instrumentation et de modélisation des données ont permis de pousser la sensibilité et la résolution spectrale atteintes aux limites fondamentales régies par l'interaction des rayons $\mathrm{X}$ 


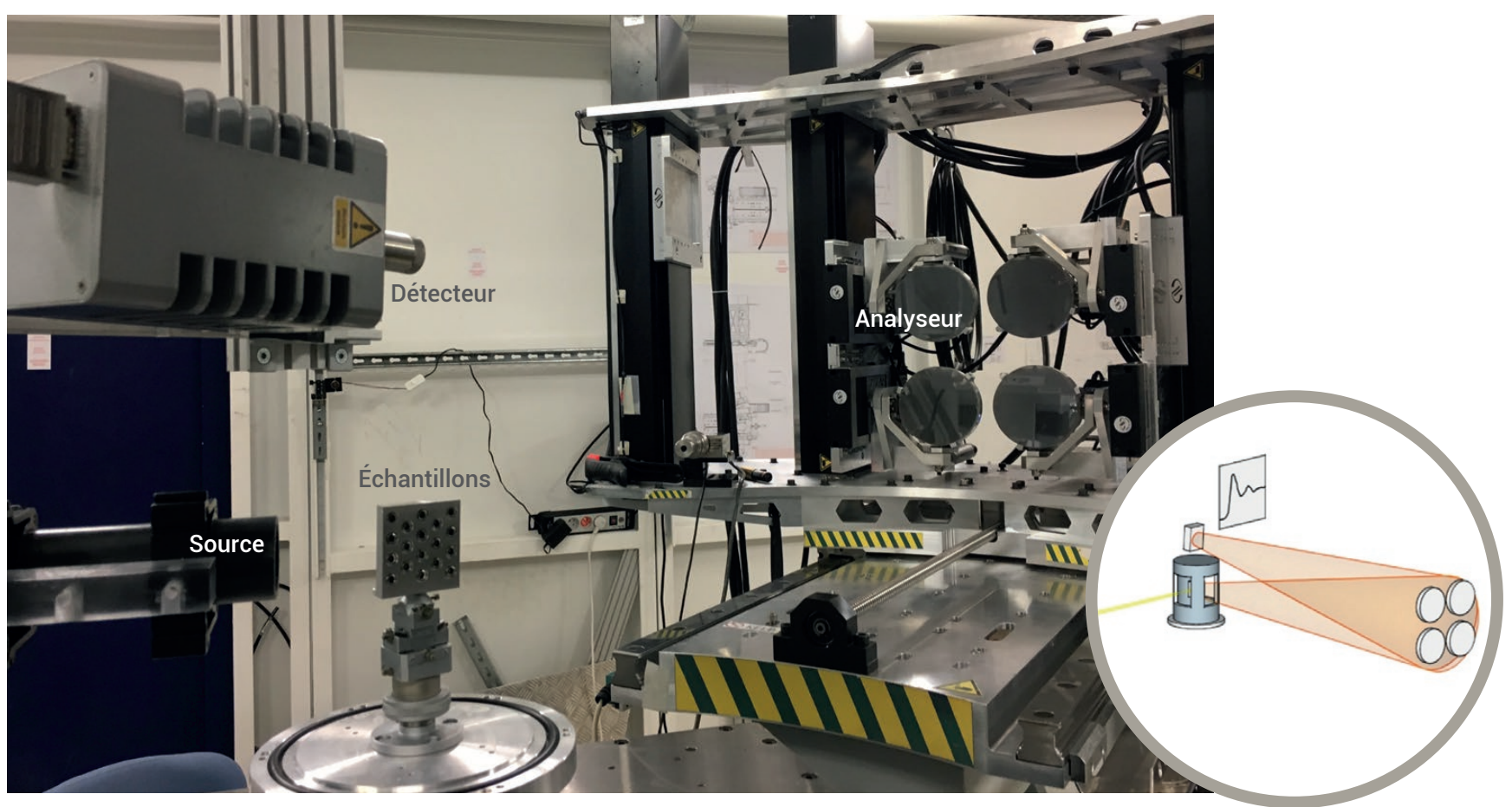

1. Exemple de dispositif d'analyse par diffusion Raman des rayons $\mathbf{X}$ (ici sur la ligne GALAXIES du synchrotron SOLEIL) et schéma de principe. Le faisceau diffusé est détecté par l'intermédiaire d'un analyseur à cristaux (ici, à quatre cristaux) qui permet de sélectionner précisément l'énergie des rayons $X$ détectés. Il est en effet essentiel de connaitre précisément la perte d'énergie dans l'échantillon pour déterminer sa composition, ce qui requiert une grande précision dans la définition des énergies des rayons $X$ incidents et des rayons $X$ détectés.

avec la matière. Cependant, la faible énergie de liaison des électrons des couches internes des éléments les plus légers du tableau périodique (jusqu'au soufre) pose une limite fondamentale à leur étude en spectroscopie XANES. Cette énergie se trouve dans la gamme dite des rayons X "mous ", entre la centaine d'électronvolts (eV) et quelques kiloélectronvolts $(\mathrm{keV})$ : le carbone a un seuil d'absorption des rayons $\mathrm{X}$, seuil d'éjection des électrons 1s, vers seulement 280-320 électronvolts, une énergie trente fois plus faible que celle du cuivre $[1,2]$. Or, la forte absorption des rayons X par la matière dans cette gamme impose des contraintes expérimentales particulièrement fortes. À ces énergies, les rayons X ne peuvent traverser plus de quelques centaines de nanomètres de matière, cent fois moins que le diamètre d'un cheveu!

\section{Diffusion Raman de rayons $X$}

Pour surmonter la limitation imposée par les rayons X mous, des rayons X durs plus pénétrants peuvent être employés avec une approche connue sous le nom de «diffusion Raman de rayons X » [3]. Ici, le processus est non résonnant : plutôt qu'être absorbés, les rayons X sont diffusés de façon inélastique, cédant une petite partie de leur énergie aux électrons $1 \mathrm{~s}$ du carbone (perte d'énergie) et contribuant à les envoyer dans des niveaux électroniques inoccupés.

Avec une telle approche, mise en œuvre de manière efficace sur de nouveaux instruments utilisant le rayonnement synchrotron, il est donc possible d'obtenir un spectre d'absorption à des énergies de quelques centaines d'électronvolts, tout en envoyant sur l'échantillon des rayons $\mathrm{X}$ "moyens » ou "durs " à des énergies beaucoup plus élevées, de plusieurs kiloélectronvolts (fig. 1). À ces énergies, les rayons $\mathrm{X}$ peuvent traverser jusqu'à plusieurs millimètres de matière ; il est alors possible d'étudier bien au-delà de leur surface la spéciation chimique de systèmes anciens [4, 5]. Le spectre obtenu peut être analysé pour identifier des absorptions caractéristiques des différents groupements chimiques contenant du carbone composant l'échantillon. La décomposition mathématique du spectre permet d'identifier les teneurs relatives de ces groupements.

La limitation principale à l'utilisation de cette spectroscopie est la faible efficacité de ce processus physique non résonnant et la difficulté expérimentale à détecter efficacement les rayons $\mathrm{X}$ diffusés. Les premiers travaux ont donc porté sur des composés organiques très purs, comme ceux issus de coupes pétrolières. Néanmoins, au cours de la dernière décennie, l'utilisation de faisceaux intenses de rayons $\mathrm{X}$ provenant de sources synchrotron modernes et les progrès de la spectrométrie des rayons $\mathrm{X}$, ont permis d'envisager l'emploi de cette méthode pour la spéciation des éléments légers dans une bien plus large gamme de systèmes et de conditions. L'étude de matériaux complexes est devenue possible. Plusieurs matériaux anciens tels que des pigments d'artistes et des macro-restes biologiques provenant de fouilles paléontologiques ont récemment pu être étudiés par cette spectroscopie. Nous en donnons ici deux exemples. 

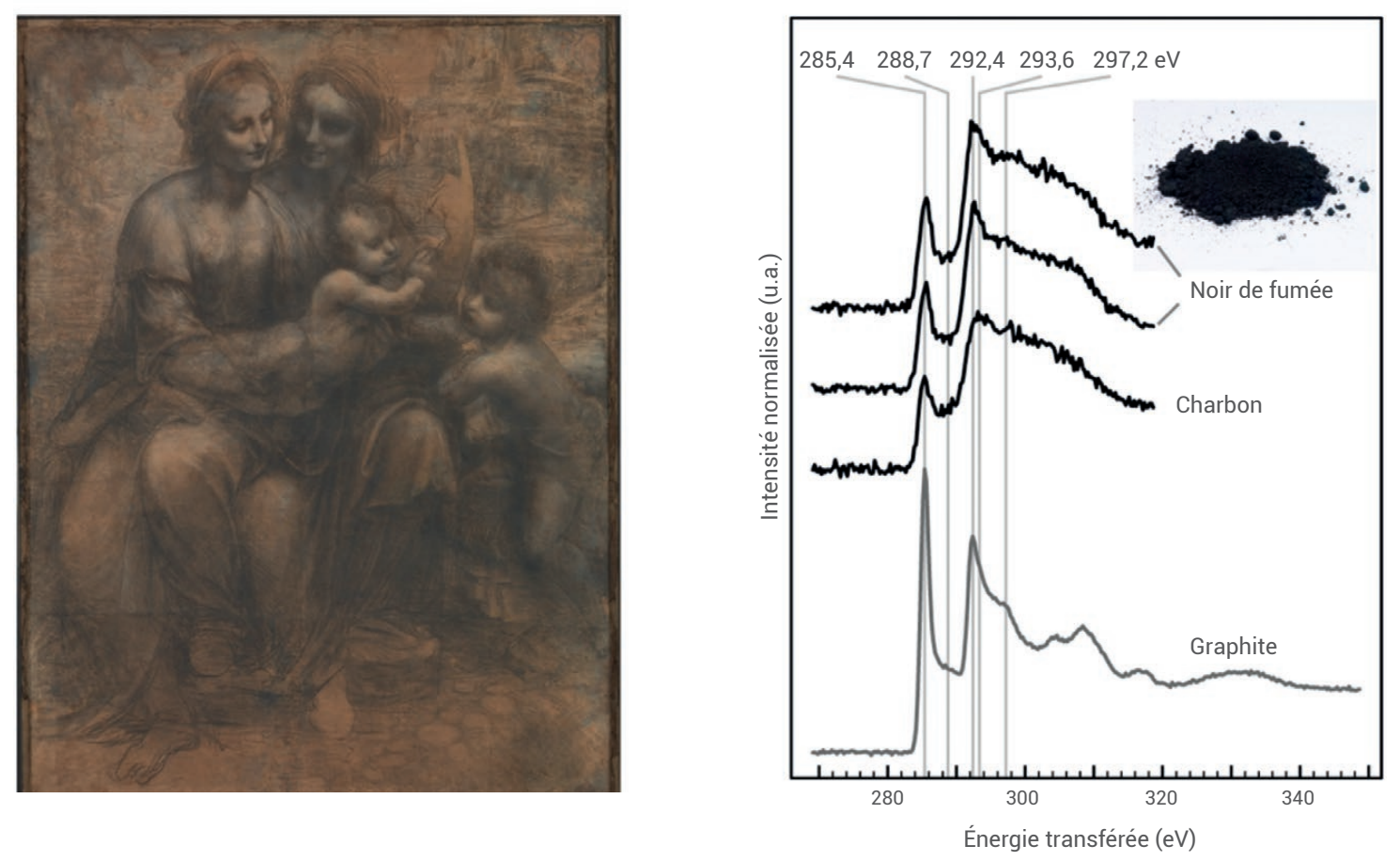

2. Études de pigments noirs de carbone. À droite : la diffusion Raman des rayons X permet de différencier des pigments noirs de carbone difficiles à identifier. À gauche, le carton de Léonard de Vinci, La Vierge, l'Enfant Jésus avec sainte Anne et saint Jean Baptiste, daté environ du milieu du $16^{\mathrm{e}}$ siècle et conservé à la National Gallery de Londres, est un exemple-type d'œuvre réalisée à partir de pigments noirs de carbone (fusain sur papier).

$>>$

\section{Encres d'artistes}

La diffusion Raman de rayons X a été employée pour différencier des encres anciennes à base de carbone employées par les artistes. La méthode fournit des informations sur la nature des liaisons chimiques liant le carbone à d'autres atomes de carbone ou à d'autres éléments chimiques dans de tels systèmes. Les charbons anthropiques (charbon, cokes, suie, etc.), tout comme ceux naturels (charbons, kérogènes dont les schistes bitumineux et gazeux) résultent de processus de carbonisation des précurseurs organiques. Au cours de ces processus, des groupes oxygénés et hydrogénés sont libérés et les produits résultants, appelés " carbonisats " (ou plus communément chars en anglais), deviennent des carbones aromatiques, c'est-à-dire dont les atomes de carbone sont organisés, plus ou moins parfaitement, en plans atomiques avec une structure de cycles hexagonaux, comme c'est le cas de façon idéalement périodique pour le graphite parfait. En fonction de la nature des précurseurs organiques et des conditions de carbonisation, on obtient des organisations multi-échelles très différentes (très éloignées de l'ordre cristallin du graphite) qui constituent une signature des procédés de fabrication [6]. Il a ainsi été possible d'étudier le degré d'aromaticité (la part de carbone engagé dans des composés formant des structures cycliques), les signatures des groupes carboxylates (COO), la présence de carbone lié à des hétéroatomes et la turbostraticité (l'organisation du graphite sous forme de plans imparfaitement parallèles les uns aux autres). La sensibilité de la méthode était suffisamment élevée pour distinguer les pigments de noir de carbone obtenus par combustion ou pyrolyse de gaz ou d'huile d'un charbon de bois (fig. 2)

\section{Carbones préhistoriques}

En paléontologie, la diffusion Raman de rayons $\mathrm{X}$ a été employée pour analyser un fragment de peau du fameux mammouth laineux de Liakhov, vieux de 49000 ans et exposé dans la galerie d'Anatomie comparée et de Paléontologie du Muséum national d'Histoire naturelle de Paris. Ce mammouth, découvert dans le permafrost sibérien en 1908 et offert à la France quatre années plus tard, est le seul spécimen de mammouth sibérien exposé dans un musée occidental. La diffusion Raman de rayons X révèle une conservation exceptionnelle de composés organiques. La caractéristique principale des spectres obtenus (fig. 3) est la présence d'un pic intense et large à $288,7 \mathrm{eV}$. Ce dernier, de forme asymétrique, est attribué à une superposition de composés carboxyles $(\mathrm{COO} / \mathrm{COOH})$ à $288,7 \mathrm{eV}$, et amides $(\mathrm{N}-\mathrm{C}=\mathrm{O}$, responsables des liaisons peptidiques formant les protéines) à $288,3 \mathrm{eV}$. La conservation de matériaux protéiques est également mise en évidence par un épaulement bien perceptible à $287,2 \mathrm{eV}$, caractéristique des liaisons carbone-soufre de certains acides aminés présents en quantité dans la peau. Le petit pic observé à $285,4 \mathrm{eV}$, typique des liaisons $\mathrm{C}=\mathrm{C}$ des carbones aromatiques, indique que les composés carbonés du fragment de peau sont très faiblement aromatisés (fig. 3). Enfin, l'absence de signaux à 290,3 et $300 \mathrm{eV}$, propres aux carbonates, confirme que la peau du mammouth n'a pas été minéralisée au cours du temps.

En fait, malgré les 49000 ans de la peau du mammouth les spectres obtenus apparaissent très similaires à ceux rapportés pour des tissus modernes (comme des tendons). Ils témoignent de l'incroyable préservation des tissus du mammouth de Liakhov jusqu'à l'échelle atomique. 

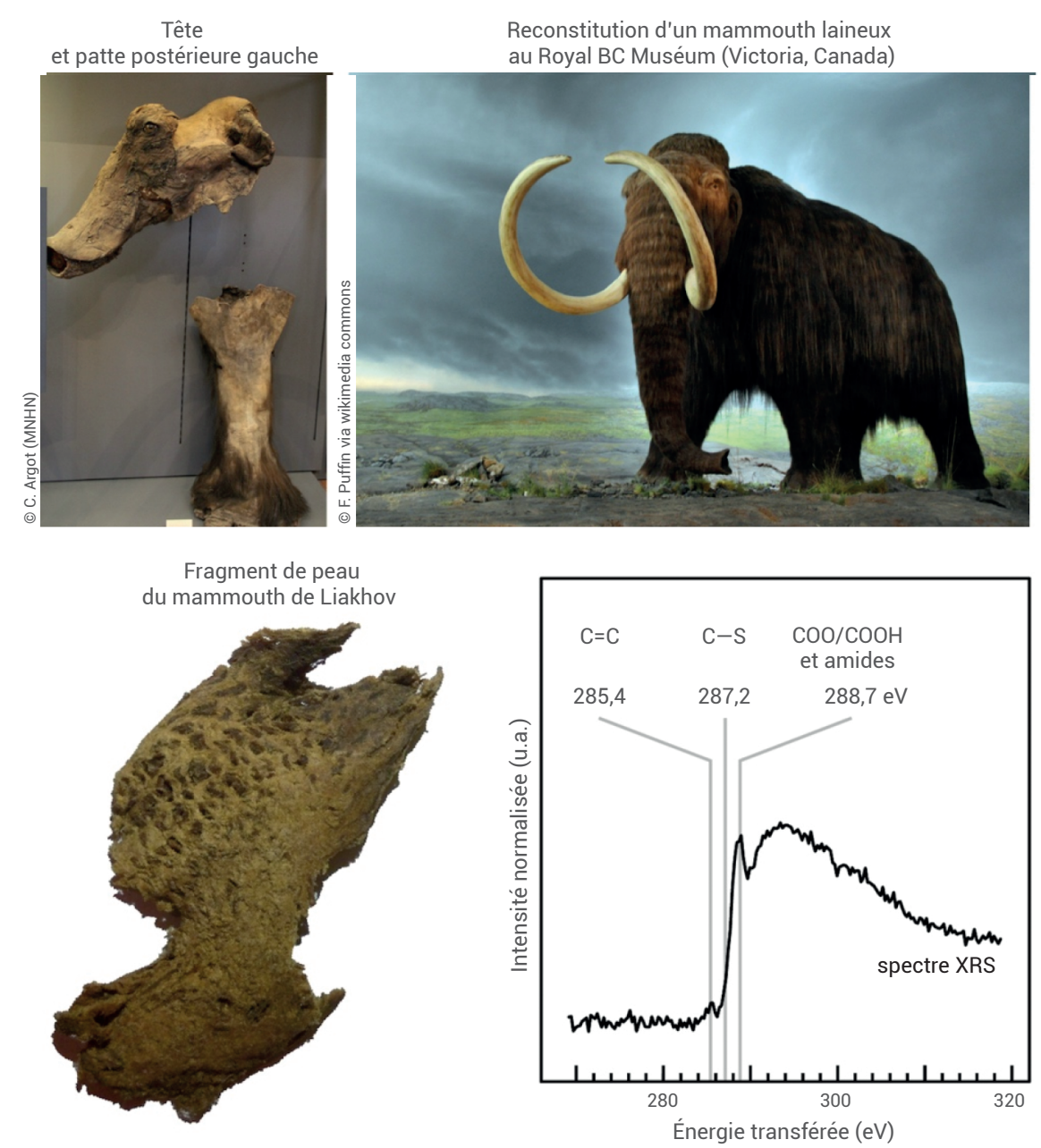

3. L'étude par diffusion Raman des rayons X (XRS) d'un fragment de peau du mammouth laineux de Liakhov, vieux de $\mathbf{4 9} \mathbf{0 0 0}$ ans, montre le fascinant état de conservation des composés organiques qui le constituent. Le spectre mesuré montre les absorptions caractéristiques d'un tissu protéique, avec des rapports d'intensité similaires à ceux observés dans un tissu moderne $[4,5]$.

\section{Références}

1 S. Bernard et D. Papineau, "Graphitic carbons and biosignatures", Elements, 10 (2014) 435-440

2• L. Bertrand et al., Top. Curr. Chem., 374:7 (2016).

3. Y. Mizuno et Y. Ohmura, "Theory of X-ray Raman scattering", Journal of the Physical Society of Japan, 22(2) (1967) 445-448.

4• P. Gueriau et al., Anal. Chem., 89 (2017) 10819-10826.

5• P. Gueriau et al., dans Synchrotron SOLEIL Highlights 2017, pp. 80-81, SOLEIL synchrotron (2018).

6• J.-N. Rouzaud et al., Comptes Rendus Geosciences, 347 (2015) 124-133.

7• J. Stöhr, NEXAFS spectroscopy, Springer Series in Surface Sciences (1992).

8• U. Bergmann et al., Microchemical Journal, 71 (2002) 221-230.

9- U. Bergmann et O. C. Mullins, dans Asphaltenes, Heavy Oils, and Petroleomics, pp. 139-155, Springer (2007).

10• P. Wernet et al., Science, 304 (2004) 995-999.

11 • R. Georgiou et al., Science Advances, 5, 8 (2019) eaaw5019.

\section{Potentiels futurs}

On sait depuis longtemps que la spectroscopie XANES est une sonde très sensible et performante de la structure locale et de la spéciation du carbone [7]. Cependant, le besoin de rayons $\mathrm{X}$ de faible énergie a restreint cette approche à une utilisation sous vide, avec une très faible profondeur de pénétration. L'utilisation de la diffusion Raman de rayons $\mathrm{X}$ durs pour la spéciation d'éléments légers s'est révélée faisable lorsque de puissantes sources synchrotron sont devenues disponibles [8], et cette technique a été appliquée à plusieurs systèmes carbonés (voir, par exemple, Bergmann et al. [9]). Elle a, jusqu'à présent, surtout été employée pour étudier des coupes pétrolières ou la structure de l'eau [10].

Les travaux rapportés ici montrent le potentiel inattendu de la diffusion Raman de rayons $\mathrm{X}$ pour discriminer des composés à base de carbone dans des échantillons anciens complexes et très hétérogènes. La méthode est ainsi applicable à des composés organiques utilisés en peinture (colorants ou pigments comme nous l'avons montré, mais également liants ou supports), à des papiers ou parchemins, ou bien encore à des spécimens d'histoire naturelle quasiment exclusivement constitués de composés organiques, comme des ambres fossiles. La concentration dans l'échantillon en éléments plus lourds qui absorbent fortement les rayons $\mathrm{X}$, tels que la plupart des métaux, constitue la principale limitation de cette méthode. Un avantage supplémentaire est que cette dernière n'est pas soumise aux règles de sélection des processus d'absorption; elle fournit donc des signatures plus riches pour identifier les composés organiques. Cette approche est applicable à une large gamme de matériaux organiques anciens et historiques. La diffusion Raman de rayons $\mathrm{X}$ n'atteint pas une résolution nanométrique, mais sonde le matériau en volume en s'affranchissant des contaminations en surface. Il est par là-même possible d'envisager l'étude d'objets organiques anciens en 3D sur des volumes millimétriques, tout en établissant leur composition en chaque point, ce qui est aujourd'hui impossible à partir de toute autre spectroscopie photonique [11]. Cette méthode semble donc promise à un avenir particulièrement brillant pour les matériaux anciens et patrimoniaux. 